\title{
ATIVIDADES VIRTUAIS PARA IMPULSIONAR A FORMAÇÃO HOLÍSTICA DE ESTUDANTES DE ENGENHARIA DURANTE O ISOLAMENTO SOCIAL DE 2020
}

Camilla Schettino Oliveira - camilla.schettino@engenharia.ufjf.br

Universidade Federal de Juiz de Fora

Rua Santo Antônio, 1005/1002

36016-210 - Juiz de Fora - Minas Gerais

Esteban Vicente Aguilar Bojorge - esteban.aguilar@engenharia.ufjf.br

Universidade Federal de Juiz de Fora

Rua Severino Meireles, 325/602

36025-040 - Juiz de Fora - Minas Gerais

Júlia Bittencourt Garcia Silva - julia.bittencourt@engenharia.ufjf.br

Universidade Federal de Juiz de Fora

Rua Espírito Santo, 1245/704

36016-200 - Juiz de Fora - Minas Gerais

Resumo: $O$ atual isolamento social tem forçado a comunidade acadêmica, bem como a sociedade, a se reinventar e se adequar à nova realidade. Este artigo relata as atividades virtuais desenvolvidas pelo Programa de Educação Tutorial da Engenharia Elétrica (PET Elétrica) da Universidade Federal de Juiz de Fora (UFJF) no intuito de complementar a formação acadêmica em engenharia e de fomentar o desenvolvimento de competências transversais. É apresentado o projeto Spa Tecnológico, mantendo um foco principal em uma de suas vertentes, o PET English, adotados como soluções para manter as atividades da equipe neste momento de incertezas. $O$ trabalho finaliza apresentando os resultados obtidos, além de uma perspectiva para o período pós-pandemia.

Palavras-chave: Isolamento social. Programa de Educação Tutorial. Competências transversais. Lingua inglesa. PET English. 


\section{INTRODUÇÃO}

O Programa de Educação Tutorial (PET), criado em 1979 pela Secretaria de Educação Superior (SESu), vinculada ao Ministério da Educação e Cultura (MEC), tem o objetivo de desenvolver projetos baseados na tríade de Pesquisa, Ensino e Extensão com o intuito de complementar a formação acadêmica de discentes, auxiliados por um professor tutor, participantes da equipe e de todo o curso de graduação apoiados pelo PET.

O PET da Engenharia Elétrica (PET Elétrica) da Universidade Federal de Juiz de Fora (UFJF) foi fundado em 1991. Na sua criação, o curso de Engenharia Elétrica era unificado. A partir de 2009, com o programa de Reestruturação e Expansão das Universidades Federais (REUNI), os cursos foram subdivididos em cinco habilitações: Energia, Robótica e Automação Industrial, Sistemas Eletrônicos, Sistemas de Potência e Telecomunicações. Todos eles são contemplados pelo PET Elétrica.

A equipe do PET consta de 18 alunos cadastrados na plataforma Sistema de Gestão do Programa de Educação Tutorial (SIGPET), sendo 12 bolsistas, 6 voluntários oficiais, além do professor tutor. Devido à alta adesão dos alunos do curso com o programa, também são aceitos voluntários não-oficiais, sejam egressos ou demais alunos alcançados pelas atividades do PET.

O PET Elétrica desenvolve trabalhos pautados nos três pilares educacionais. Seu objetivo é ser o elo entre o conhecimento científico e a sociedade, levando até ela conhecimentos sobre ciência, tecnologia e inovação em Engenharia Elétrica. Adicionalmente, são incentivadas práticas em que o aluno aprende através de atividades nas quais é possível reforçar conhecimentos já adquiridos na Faculdade e também são fomentadas atividades de pesquisa. Atualmente são desenvolvidas atividades de Ensino, como o projeto Linusbot, uma competição de robôs seguidores de linha para calouros da Engenharia Elétrica; atividades de Pesquisa, como a Impressora 3D construída por alunos do PET, e atividades de Extensão, entre elas o portal de notícias Energia Inteligente. Os membros do PET Elétrica UFJF participam em mostras, eventos e congressos e desenvolvem atividades de formação, como o Student Professional Awareness Conference (SPAC), que são palestras de profissionais na área de engenharia que abordam suas experiências de trabalho, sem foco em aspectos técnicos. Durante os recessos escolares, também é desenvolvida a atividade Spa Tecnológico, que consiste no desenvolvimento de minicursos, visitas técnicas e integrações culturais.

No ano de 2020, frente a pandemia do novo coronavírus (Sars-CoV-2), o programa precisou se reinventar para continuar desenvolvendo seus projetos de maneira remota, visto a paralisação das atividades presenciais na universidade e consequentemente das atividades do PET são, majoritariamente, realizados na sala do programa. Diante desse cenário, o PET Elétrica decidiu desenvolver as atividades do projeto Spa Tecnológico, que ocorre de segunda à sexta-feira, na modalidade remota. A denominação de Spa Tecnológico se faz por meio de uma metáfora com um spa, em que o tratamento é focado na saúde e no bem estar físico e mental, porém, para o PET Elétrica, o tratamento se baseia em novos conhecimentos em engenharia e tecnologia. No cronograma do Spa Tecnológico estão inclusas as atividades do projeto PET English, que é de extrema relevância profissional e pessoal ao permitir o desenvolvimento da língua inglesa por parte dos membros do PET Elétrica ("petianos").

Este trabalho visa relatar as experiências vivenciadas pelo grupo PET Elétrica UFJF com o projeto PET English, no âmbito do Spa Tecnológico, durante o isolamento social. 


\section{PROJETOS}

Nesta seção será contextualizado o que são o Spa Tecnológico e o PET English, além de relatar as adaptações para o período de isolamento social.

\subsection{Spa Tecnológico}

O Spa Tecnológico é um projeto focado na difusão do conhecimento entre os petianos por meio de minicursos, bem como na aplicação da metodologia central na qual é fundado o PET, o Project Based Learning. Isso possibilita aprimorar a capacidade colaborativa e de trabalho em equipe, além de proporcionar momentos de reflexão sobre a estrutura da educação em âmbito local, reflexão sobre temas que permeiam a equipe, além de momentos culturais e de integração dos petianos.

Após o início da pandemia, foi elaborada uma programação diferente para cada semana, completamente remota. Inicialmente, o Spa foi um momento de capacitação interna do grupo, no qual foram ministrados minicursos de modelagem 3D usando o software Fusion, desenhos gráficos no CorelDRAW e Canva, programação nas linguagens Html, CSS e JavaScript, prototipagem de placas de circuito impresso no software Proteus e desenvolvimento de currículos online na plataforma LinkedIn. Outras atividades desenvolvidas envolveram o compartilhamento de experiências profissionais, exposição sobre área de atuação e pesquisa e conselhos aos futuros engenheiros pelos egressos do programa, professores da Faculdade e profissionais do ramo de Engenharia. Além disso, foram concebidas atividades de cunho cultural, como apresentações de temas como folclore brasileiro e uma sessão musical entre os membros. Por último, foram realizados encontros com outros grupos PET de todo o Brasil, para conhecer suas realidades e trocar.

\subsection{PET English}

O projeto PET English fomenta o aprendizado e a prática da língua inglesa em seus quatro pilares: speaking (fala), writing (escrita), reading (leitura) e listening (audição). Antes da pandemia, era de comum acordo que, em um dia predeterminado da semana, em reunião do PET só fossem permitidas conversas em inglês e fossem realizados exercícios para aprimorar o vocabulário e a gramática. Um desses exercícios era o Vocabulary of the day, no qual era escolhida uma palavra curiosa para enriquecer o conhecimento.

O projeto é conduzido por três petianos com o nivel de Inglês avançado que possuem certificados de proficiência internacional, tais como Key English Test (KET), Preliminary English Test (PET) e First Cambridge Exam (FCE), fornecidos por Cambridge, e o Test of English as a Foreign Language (TOEFL) Institutional Testing Program (ITP), oferecido pela UFJF. Os demais participantes da equipe, em sua maioria, não realizaram cursos formais de inglês e por isso apresentam niveis de conhecimentos diversificados.

Devido à realidade de uma equipe grande e desnivelada em relação aos conhecimentos da língua, a comissão coordenadora do PET English idealizou novas atividades para estimular o interesse dos petianos pela língua, mesmo durante o isolamento social.

Os encontros foram divididos em quatro vertentes: PET English Movie, PET English Invites, PET English Writing e PET English Gathers, que diversificam as atividades dentro de uma só proposta, buscando atingir mais petianos.

\section{METODOLOGIA}

Logo após o início do período de isolamento social, a equipe coordenadora passou a se reunir semanalmente para discutir e decidir as ações que seriam adotadas para as atividades relacionadas com o PET English. O PET English Movie foi realizado através de encontros 
semanais para a discussão de filmes, documentários e séries cujos temas permitissem reflexões sobre a sociedade ou a engenharia. Com este roteiro foram realizadas diversas perguntas aos petianos para guiar o debate. Alguns títulos de filmes abordados foram: "A Viagem de Chihiro", "Sérgio", "O menino que descobriu o vento", "A onda", "Sociedade dos poetas mortos" e "Parasita", que possibilitaram discussões pertinentes sobre ganância social, conflitos geopolíticos, pobreza, desigualdade social, reinvenção tecnológica, neonazismo, vertentes políticas e educação libertadora.

O PET English Invites se encarregou de contatar profissionais de outros países, de diversas áreas, para apresentar virtualmente aos petianos, desde seu âmbito profissional, suas áreas de atuação e até um pouco da sua cultura. Os alunos também apresentaram para os convidados o perfil do grupo PET, a Faculdade de Engenharia da UFJF, a Universidade e atrações turísticas da Cidade de Juiz de Fora, além do panorama geral do país. As perguntas feitas aos convidados ao final das apresentações foram elaboradas com antecedência, de acordo com seus campos de atuação. Os encontros foram realizados através da plataforma Google Meet e permitiram conhecer a vivência de um estudante de bacharelado na Califórnia, envolvendo as questões financeiras, sociais e educacionais, na perspectiva de um professor e pesquisador universitário, o $\mathrm{PhD}$. Tom Huxford. Também foi possível aprender sobre a realidade feminina nas Ciências Exatas e na Engenharia com a PhD. Ritu Raman e a preocupação social inerente aos projetos desenvolvidos em um dos maiores institutos de tecnologia do mundo, o Massachusetts Institute of Technology (MIT). Por fim, foi apresentada a estrutura organizacional de uma empresa multinacional, como a Toyota com o Vice-Diretor Comercial, o engenheiro Patricio Sarabia, quem discorreu sobre metodologias para resolução de problemas corporativos em nível global.

O PET English Writing consistiu na atribuição de tarefas aos petianos para que pudessem desenvolver sua fluência na escrita. Foram explorados diferentes gêneros textuais para que se tornassem capazes de se comunicar em situações diversas.

O PET English Gathers realizou encontros com outros grupos PET do Brasil, discutindo, em inglês, a organização interna dos grupos, seus projetos e atividades. Além de permitir conhecer a realidade de grupos similares, foram debatidos temas que aproximam diferentes áreas do conhecimento como, por exemplo, a filosofia educacional na qual os discentes estão inseridos e como isso impacta nas estruturas familiares e os conflitos protagonizados pelos petianos, tendo como pano de fundo os filmes discutidos no projeto. Em um segundo momento, a equipe do PET desenvolveu atividades em inglês, apresentando novo vocabulário, sanando dúvidas e realizando, de fato, uma integração. Foram abordadas questões de cunho pessoal, que permitiram aos petianos se conhecer melhor e fortalecer sua consciência individual ante a sociedade.

O desempenho das atividades desenvolvidas foi avaliado mediante uma pesquisa entre os integrantes da equipe, cujos resultados e análises são apresentadas na seguinte seção.

\section{RESULTADOS}

A equipe coordenadora, esperando compreender melhor os resultados e impactos obtidos com o desenvolvimento do projeto de melhoria da língua inglesa e incentivo à sua prática, aplicou um questionário avaliativo através do Google Forms, abrangendo os tópicos citados na seção anterior, referentes às atividades do projeto em si, mas também à motivação pessoal de cada petiano, o qual foi respondido por todos os participantes.

Primeiramente, os principais pontos para análise são referentes às motivações quanto à participação. Foram obtidas 14 respostas que resultaram nas figuras 1 e 2, em relação às perguntas: "Qual a sua motivação para participar do projeto inicialmente?" e "Qual a sua motivação para participar do projeto agora?”, respectivamente. 
(C) COBENCE

"Os desafios para formar hoje o engenheiro do amanhã"

Figura 1 - Gráfico circular para "Qual a sua motivação para participar do projeto inicialmente?”.

Qual a sua motivação para participar do projeto inicialmente?

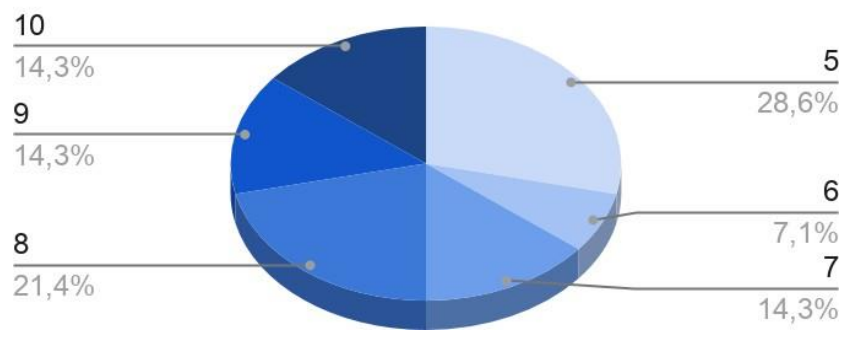

A princípio, considerando a não obrigatoriedade do projeto, a motivação foi alta, com média 7,28, o que também é perceptível pela grande adesão ao projeto, o qual abrange 17 dos 25 petianos.

Figura 2 - Gráfico circular para "Qual a sua motivação para participar do projeto agora?”.

Qual a sua motivação para participar do projeto agora?

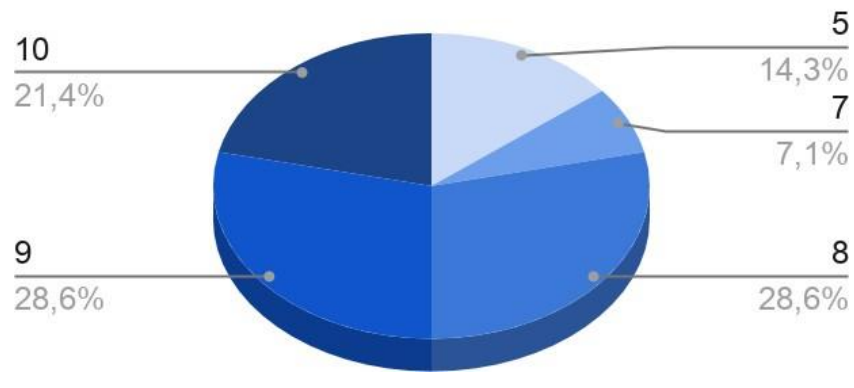

É perceptível um aumento na motivação após dez semanas de atividades, o que ilustrou uma média de 8,21 . Isso pode ser explicado pelo fato de o projeto tratar de temas relacionados não apenas à engenharia. No entanto, também pode ser visto como um momento de descontração, o que é vital, principalmente durante o isolamento por conta da pandemia.

Duas modalidades que devem ser destacadas pela melhor avaliação são: PET English Movie e PET English Invites. As figuras 3 e 4 mostram as notas obtidas para esses dois subprojetos. 
Figura 3 - Gráfico circular para "Como você avalia o PET English Movie?”.

Como você avalia o PET English Movie?

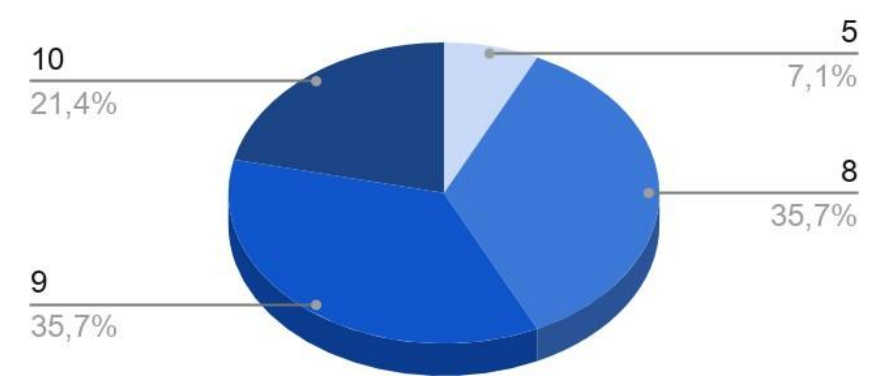

Durante o PET English Movie, o grupo relatou sentir-se mais desinibido para falar em inglês e maior interatividade cultural, visto que foram escolhidos filmes do mundo inteiro. Neste subprojeto foi praticado o listening e o speaking.

Figura 4 - Gráfico circular para "Como você avalia o PET English Invites?”.

Como você avalia o PET English Invites?

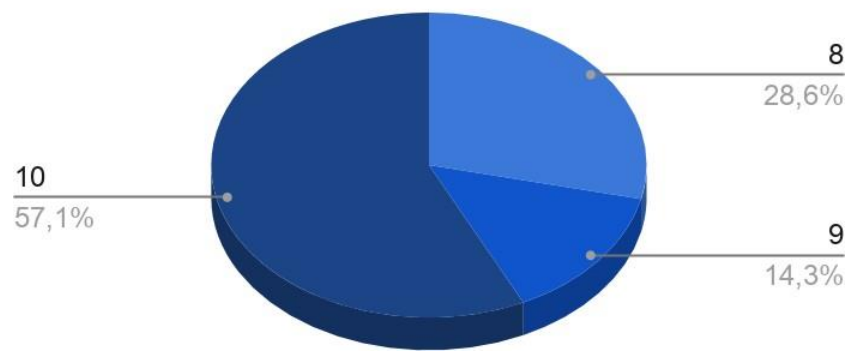

O PET English Invites demonstrou desafios diferentes aos participantes, pois, pela sua estrutura, na qual no primeiro momento, os petianos apresentaram a equipe ao entrevistado, possibilitou a interação em língua estrangeira, expandindo a zona de conforto dos petianos ao praticar o speaking; no segundo momento, o convidado falou sobre suas atividades e trajetória profissional, o que possibilitou desenvolver o listening.

\section{CONSIDERAÇÕES FINAIS}

O modelo de engenheiro demandado hoje não apenas pelo mercado, mas também pela sociedade, não é mais uma mera mão de obra altamente especializada, mas um cidadão pleno. À medida que o mundo se torna mais complexo, os engenheiros devem apreciar, mais do que nunca, as dimensões humanas da tecnologia, ter uma noção de questões globais, ser sensíveis à diversidade cultural e saber como se comunicar de maneira eficaz (BORGES, R.L, 2013). Em suma, eles devem ser muito mais versáteis do que o estereótipo tradicional do nerd social.

As chamadas soft skills ou competências transversais são extremamente importantes para o desenvolvimento da carreira profissional bem sucedida do engenheiro. Elas abrangem uma comunicação eficaz, pensamento criativo e crítico, resiliência, empatia, liderança, além de ética no trabalho e proatividade (DI SPAGNA, J, 2017). Com essa nova visão de formação, o 
conhecimento de língua estrangeira, em especial da língua inglesa deixou de ser um diferencial e, sim, uma exigência.

A concepção de formação holística do engenheiro vai de encontro com a filosofia de criação dos grupos PET, que espera proporcionar a melhoria da qualidade acadêmica dos cursos de graduação apoiados, referidos no Manual de Orientações Básicas do Programa de Educação Tutorial - PET (MINISTÉRIO DA EDUCAÇÃO; SECRETARIA DE EDUCAÇÃO SUPERIOR, 2006):

"As atividades extracurriculares que compõem o Programa têm como objetivo garantir aos alunos do curso oportunidade de vivenciar experiências não presentes em estruturas curriculares convencionais, visando a sua formação global e favorecendo a formação acadêmica, tanto para a integração no mercado profissional quanto para o desenvolvimento de estudos em programas de pós graduação."

As cadeiras de formação científica ofertadas pela universidade são uma base para o desenvolvimento das habilidades técnicas de um engenheiro, no entanto para o PET Elétrica UFJF, o projeto PET English é tão essencial quanto os projetos de viés técnico do grupo.

O PET English fornece a oportunidade do contato mais direto com a língua inglesa para os petianos, sem a necessidade de um curso paralelo, de maneira mais descontraída e informal, familiarizando a todos com jargões da área tecnológica e do cotidiano, que também são divulgados semanalmente através das redes sociais do grupo.

Com o isolamento social os projetos do PET tiveram que encontrar soluções de acordo com o novo cenário de isolamento. Não se sabe como será o mundo ao findar da pandemia, mas há grande chance de que a nova "normalidade" venha a ser diferente daquela à qual o mundo estava acostumado. Com isso, o PET English deverá se reinventar a fim de se adaptar às novas condições e desafios e, assim, continuar a cumprir com sua missão.

No pós-pandemia, pretende-se reforçar os encontros semanais com os membros do grupo, porém adequando à realidade de aulas presenciais e atividades integrais. Serão sempre abordadas discussões sobre temas pertinentes à sociedade, artigos científicos e inovações tecnológicas. O projeto também continuará desenvolvendo seus exercícios de escrita através do PET English Writing, ampliando os conhecimentos em gramática e vocabulário.

Quanto ao Spa Tecnológico, suas atividades presenciais serão retomadas nos períodos de férias letivas da UFJF, incluindo também momentos de práticas esportivas e de integração entre os petianos.

Portanto, sabendo da importância de um engenheiro-cidadão, capaz de construir hoje uma sociedade melhor desenvolvida econômica e socialmente, os projetos PET English e Spa Tecnológico mostram-se como atividades eficazes para complementar e suplementar a formação do acadêmico de engenharia.

\section{Agradecimentos}

Ao Programa de Educação Tutorial PET/MEC, à Faculdade de Engenharia da Universidade Federal de Juiz de Fora e aos entrevistados pelo suporte para realização deste trabalho. 


\title{
REFERÊNCIAS
}

BORGES, R.L. Globalização requer novas competências do engenheiro (entrevista). Disponível em: https://www.cimentoitambe.com.br/massa-cinzenta/globalizacao-requernovas-competencias-do-engenheiro/. Acesso em: 06 jul. 2020.

DI SPAGNA, J. 6 "Soft Skills" mais requisitadas pelo mercado. Disponível em: https://forbes.com.br/carreira/2017/07/6-soft-skills-mais-requisitadas-pelo-mercado/. Acesso em: 07 jul. 2020.

\section{MINISTÉRIO DA EDUCAÇÃO; SECRETARIA DE EDUCAÇÃO SUPERIOR. Programa} de Educação Tutorial - PET - Manual de Orientações Básicas. Disponível em: http://portal.mec.gov.br/index.php?option=com_docman\&view=download\&alias=338manualorientabasicas\&category_slug=pet-programa-de-educacao-tutorial\&Itemid=30192. Acesso em: 05 jul. 2020.

\section{VIRTUAL ACTIVITIES TO STIMULATE UNDERGRADUATE ENGINEERING STUDENTS IN THEIR HOLISTIC QUALIFICATION DURING SOCIAL DISTANCING IN 2020}

\begin{abstract}
The current social distancing policy has forced the academic community, as well as the society, to reinvent and fit into the new reality. This paper reports the virtual activities developed by Programa de Educação Tutorial of Electrical Engineering (PET Elétrica) of Universidade Federal de Juiz de Fora (UFJF) on the context of an additional engineering undergraduation and to foster soft skills development. It will be presented the project Spa Tecnológico, focusing on the correlated project, PET English, which will be detailed hereinafter as solutions found to keep the group's activities during this moment of uncertainties. This paper ends presenting the positive obtained results and a follow-up suggestion for the post-pandemic period.
\end{abstract}

Keywords: Social distancing, Soft Skills, Programa de Educação Tutorial, English language, PET English. 